\title{
And then there was light
}

\author{
It's finally happened. With the first detection of a neutron star merger by LIGO and Virgo, astronomers have at long \\ last begun the exploration of multi-messenger gravitational-wave astrophysics.
}

$\mathrm{N}$ ot everybody has a firm grasp of general relativity or black hole mergers, but the Laser Interferometer Gravitational-Wave Observatory (LIGO) brought these concepts prominently into our collective consciousness with the first detection of gravitational waves in 2015 . Announced in early 2016, their discovery changed the course of modern astrophysics research. LIGO achieved the first direct detection of a black hole (or two). We could 'hear' the Universe in its cosmic past, and the phrase 'ripples in spacetime' has entered our common parlance, 100 years after Albert Einstein predicted them. And on 17 August 2017, counterpart electromagnetic signals accompanied the gravitational waves and multi-messenger gravitational-wave astrophysics was born.

This year's Nobel Prize in Physics was awarded to Rainer Weiss, Barry Barish and Kip Thorne "for decisive contributions to the LIGO detector and the observation of gravitational waves". If it seems a fast move for the Nobel-prize committee, don't forget that Weiss first conceived of the idea back in 1967. It then gained momentum when he joined forces with Thorne in 1976, and then with Ronald Drever in 1979. These three visionaries were the founders of LIGO. But to translate that vision to a successful instrument, they needed leadership and funding for their 'blue sky' project, and that was where Barish, as director of the project, made his mark. If anything, this prize was for the perseverance and determination (as well as vision and ingenuity) of everyone involved in the LIGO collaboration, including the National Science Foundation. (See the Nature Research Collection celebrating the 2017 Nobel Prize in Physics.)

Up until the Nobel Prize announcement, each subsequent gravitational wave that LIGO, and then the LIGO Scientific and Virgo collaborations (LVC), announced with great fanfare involved mergers of black holes. However, the only signals from such mergers are gravitational waves in the frequency range from $10 \mathrm{~Hz}$ to several hundred $\mathrm{Hz}$. We could only hear but not see. Moreover, even with three detectors, the localization of a source on the sky is still many tens of square degrees. Besides adding more gravitational wave detectors, or nodes, to the network of interferometers - such as those under construction in India and Japan - we needed existing telescopes to get in on the action. Gravitational wave event GW 170817 gave astronomers exactly that opportunity.

The LVC detection of two neutron stars merging set pulses racing, as this time the emission of photons as well as gravitational waves occurred - and were detected. After receiving the alert for follow-up detections at electromagnetic wavelengths, it was hours until sunset in Chile, recalls Stefano Covino of the delay in starting the search using the European Southern Observatory/ Very Large Telescope. But fortunately, the optical counterpart, a kilonova or macronova, was localized in less than an hour, near the elliptical galaxy NGC4993. The position of the merger was lucky, he emphasizes. Most of the telescope teams were targeting the brightest galaxies within the search area defined by LVC. Gammaray bursts (GRBs) have also occurred near small and faint galaxies, and if that had been the case this time, it would have taken much longer to locate, if it were possible at all.

Astronomers have another advantage: previous experience in studying GRBs and gamma-ray afterglows. These high-energy transient events occur on timescales of milliseconds to hours to days, so astronomers are used to working in large collaborations with massive collective observational power. And this time, they were more than ready. On 16 October, concurrently with the LVC's announcement of GW 170817, more than thirty papers were published on the electromagnetic counterparts across the whole spectrum, from radio waves to infrared to visible to X-rays to gamma rays. Seven of these papers appear in Nature and Nature Astronomy. Accompanying News \& Views articles by M. Coleman Miller (Nature https://doi.org/10.1038/nature24153; 2017) and Tyler Pritchard (Nat. Astron. https:// doi.org/10.1038/s41550-017-0311-1;2017) sum up the findings. Not only do the papers support the LVC detections in an independent way, they help confirm that short GRBs and kilonovae are products of neutron star mergers and provide information on the nucleosynthesis of elements heavier than iron in the Universe. It was a collective effort to launch a new research field.
But when so many scientists are involved, an interesting sociological situation develops. How is it possible to keep a lid on the discovery? From the first LIGO detection, the collaboration has been careful to only announce peer-reviewed work. After all, David Shoemaker, spokesperson for LIGO, at a recent conference in London, UK, titled The Promises of Gravitationalwave Astronomy said that posting on Twitter is not the same as peer review. However, the simple fact of the latest neutron star detection was a badly kept secret. Indeed, when more than 70 telescopes suddenly and concertedly focus on the same patch of sky, people will wonder and it's no surprise that rumours swirled around the Internet for two months. The LVC is content that the quantitative details were able to stay under wraps until they were thoroughly vetted.

At this point, it's worth noting that the LIGO Scientific Collaboration (LSC) is not some secret society. It is an 'open' collaboration. The LIGO Data Management Plan stipulates that anyone may join and have access to LIGO data "based on their willingness to contribute to LIGO." As of October 2017, the members number more than 1,200 across more than 100 institutions. All papers authored by a member must be approved by the LSC prior to publication, a process that takes several weeks.

With such a large collaboration, the LVC open data policy is an evolving one. Indeed, a transition to open data is outlined in the Data Management Plan (in section 1.3). By the third observation run (O3; slated for 2018/2019), six-month blocks of data will be released every six months with an 18-month delay from acquisition. A significant change will be "prompt public alerts of transient events" for rapid follow-up by others. However, the extended proprietary period between acquisition and release of data seems out of step with the open nature of the collaboration. This period is a topic of active discussion within LVC. Given the wealth of experience in the team, we can be sure that the current 18-month proprietary period for data validation will shrink dramatically. It will be interesting to see new detections being delivered on a weekly basis. These are exciting times.

Published online: 2 November 2017 https://doi.org/10.1038/s41550-017-0318-7 